\title{
Avaliação da resolutividade e da satisfação da clientela de um serviço de referência secundária em of talmologia da Universidade Federal de São Paulo - UNIFESP
}

\author{
Resolution and user's satisfaction evaluation of a secondary ophthalmic reference \\ service of the Federal University of SãoPaulo-UNIFESP
}

\author{
Rosana Maura Gentil ${ }^{1}$ \\ Sandra Maria Reis Leal ${ }^{2}$ \\ Marinho Jorge Scarpi ${ }^{3}$
}

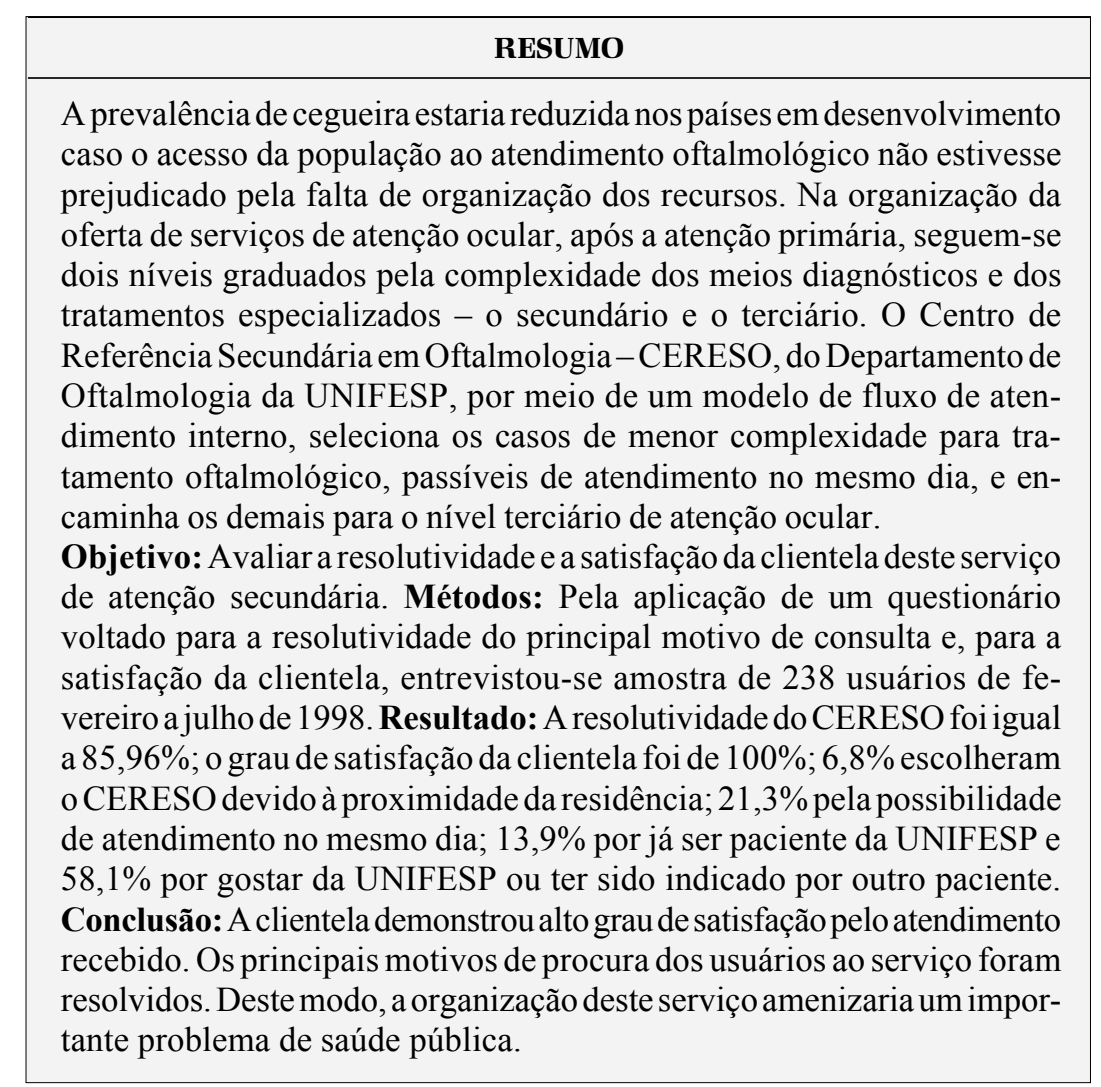

Descritores: Cegueira/prevenção \& controle; Níveis dos cuidados de saúde; Saúde ocular; Avaliação de processos e resultados (cuidados de saúde); Garantia da qualidade dos cuidados de saúde; Satisfação do paciente; Serviços de saúde; Prestação de cuidados de saúde
Médica oftalmologista e bolsista do CNPQ, especialista em Administração Hospitalar e em Serviços de Saúde pela Faculdade de Saúde Pública da Universidade de São Paulo.

2 Enfermeira do Centro de Referência Secundária em Oftalmologia - CERESO, da Universidade Federal de São Paulo - UNIFESP-EPM.

${ }_{3}^{3}$ Professor Livre Docente e Adjunto, médico oftalmologista do Centro de Referência Secundária em Oftalmologia - CERESO, da Universidade Federal de São Paulo - UNIFESP.

Esta investigação recebeu o apoio financeiro do $\mathrm{CNPq}$ - Processo $n^{\circ}$ 000524216/96

Endereço para correspondência: Rua Morcote, 47 São Paulo (SP) CEP 04023-062

E-mail: oftrosana@ig.com.br

Recebido para publicação em 21.11.2001 Aceito para publicação em 22.07.2002

Nota Editorial: Pela análise deste trabalho e por sua anuência na divulgação desta nota, agradecemos ao Dr. Jacob Moyses Cohen.

\section{INTRODUC̣̃̃O}

Os casos de cegueira distribuem-se de forma desigual e concentram-se nos países subdesenvolvidos, onde encontram-se aproximadamente 21 milhões dos 38 milhões de cegos existentes no mundo ${ }^{(1)}$.

Um dos fatores que influencia esta desigualdade é a falta de organização dos recursos para o atendimento oftalmológico, sendo que $70 \%$ dos casos de cegueira poderiam ser evitados aplicando-se conhecimentos e técnicas atualmente disponíveis, havendo uma deteç̧ão precoce e possibilitando o encaminhamento dos problemas oculares ${ }^{(1)}$. 
Em função da excessiva demanda na área de oftalmologia, associada à falta de organização dos serviços e da dificuldade de acesso do usuário ao serviço de atenção oftalmológica, estabelece-se um fluxo desordenado e vicioso no atendimento médico. Sabe-se que o maior obstáculo na atenção ocular parece não residir na falta de tecnologia adequada e sim na dificuldade de criar condições propícias para motivar pessoas, pouco acesso aos serviços, falta de infra-estrutura e de organização da assistência oftalmológica ${ }^{(2)}$.

Simeant ${ }^{(3)}$, em trabalho realizado em uma região sanitária do Chile, estudou a capacidade resolutiva da morbidade nos níveis de atenção e concluiu que as "Enfermidades do olho" são uma das primeiras causas de referência médica desde o nível primário. Notou, porém, que um grande número dos transtornos oculares correspondiam a vícios de refração, podendo-se chegar à conclusão que o poder resolutivo do nível primário para as enfermidades do olho poderia ser consideravelmente aumentado a um custo moderado, o que contribuiria em larga medida a aumentar o poder resolutivo do nível primário em geral, com benefícios óbvios aos pacientes.

Donabedian $^{(4)}$ analisa a atenção à saúde por seus três componentes: a atenção técnica, o manejo das relações interpessoais e o ambiente na qual se desenvolve o processo da atenção. A avaliação de cada um destes aspectos permite estimar a qualidade da atenção, devendo-se levar em consideração as características específicas do país em que se desenvolve este processo.

Segundo Aguirre-Gas ${ }^{(5)}$, a avaliação da qualidade da atenção médica, tem se centrado na informação contida no expediente clínico, porém deve-se levar em conta outros aspectos: a suficiência e a qualidade dos recursos físicos, a disponibilidade e a capacidade profissional e técnica dos recursos humanos (satisfatória com o nível de atenção que atuam), a administração dos materiais de consumo, a organização dos serviços, a realização dos procedimentos e técnicas segundo normas de qualidade vigentes, a obtenção de resultados da atenção em concordância com o objetivo da mesma e todo o elo social e humanitário existente. Este mesmo autor também considera a qualidade de atenção enfocada por três ângulos diferentes, conforme a satisfação das expectativas do usuário ou paciente, do profissional de saúde e da instituição.

Assim deve existir um balanço adequado entre a qualidade e a eficiência na prestação de serviços, garantindo a qualidade do atendimento através da qualidade técnica, da sua eficiência (relação custo/benefício), cobertura assistencial e da satisfação dos pacientes ${ }^{(5)}$.

A organização da atenção médica, que segue níveis de complexidade crescente, deve partir de uma análise cuidadosa da realidade e ser submetida a revisões periódicas a fim de se evitar distorções, usualmente de alto custo, no manejo global das necessidades da atenção à saúde da população ${ }^{(6-7)}$.

Segundo a OPAS ${ }^{(8)}$, a oferta de serviços de atenção ocular deve ser planejada e organizada como parte de um sistema na qual se identifiquem os problemas, se criem planos para re- solvê-los, se definam os recursos necessários para executar suas atividades e se programem as atividades e tarefas a serem realizadas, em diferentes níveis de complexidade. A hierarquização das ações de saúde e distribuição dos serviços é conhecida como atenção primária, secundária e terciária, sendo a primária conhecida como atenção básica de saúde. Dentro deste modelo considera-se que para contemplar os serviços de atenção ocular bastam dois níveis:

- nível de atenção básico;

- nível especializado.

Os serviços básicos compreendem a identificação de fatores de risco, a promoção da saúde ocular, o diagnóstico precoce e, em algumas condições, os tratamentos simples; também inclui o controle periódico das pessoas sadias e enfermas e aspectos básicos da reabilitação.

Os serviços especializados de atenção secundária e terciária, além de cobrir os aspectos básicos, devem estimular os procedimentos complexos de diagnóstico, tratamento e reabilitação.

Ambos níveis compartilham sua responsabilidade nos processos de capacitação de pessoal, supervisão de atividades, avaliação e monitoria.

Os componentes fundamentais na prestação de serviços de saúde são a avaliação e a monitoria, que servem para melhorar as condições de eficiência dos mesmos serviços, fornecendo elementos de julgamento para justificar a obtenção de recursos humanos, materiais e financeiros e facilitar o processo de tomada de decisões ${ }^{(8)}$.

Um dos resultados das recomendações do informe Flerner, de 1910, foi a grande especialização da medicina, influenciando diretamente nas instituições educativas formadoras de recursos humanos no país assim como a organização do hospital, agrupados em especialidades clínicas, determinando uma forma de atenção à população associada à utilização de tecnologia como instrumento de opção para o trabalho médico, que muitas vezes se apresenta imprescindível para a prática médica. Em outras palavras, tem se dado lugar a uma profunda fragmentação do saber médico em diversas especialidades, uma enorme tecnologização da prática médica e, conseqüentemente, uma grande despersonalização do exercício da medicina ${ }^{(9)}$.

Isto associado a um aumento da matrícula nas instituições formadoras de recursos humanos nesta área, a construção acelerada de hospitais, a intensificação do sistema de especialidades médicas, a institucionalização da medicina e com ela a burocratização da norma, levou a um desequilíbrio entre a oferta e a demanda de serviços e uma diminuição da qualidade da atenção ao paciente, principalmente com relação à dimensão interpessoal ${ }^{(9)}$.

Este estudo visa avaliar a qualidade da atenção oftalmológica no Centro de Referência Secundária em Oftalmologia (CERESO) do Departamento de Oftalmologia da Universidade Federal de São Paulo (UNIFESP) - quanto ao grau de resolutividade do serviço e ao aspecto referente à satisfação do usuário. 


\section{MÉTODOS}

Estudou-se a população usuária do Centro de Referência Secundária em Oftalmologia (CERESO) do Departamento de Oftalmologia da Universidade Federal de São Paulo (UNIFESP), localizado no Bairro de Vila Clementino, zona sul do município de São Paulo - Estado de São Paulo - Brasil.

Este centro de referência secundária faz parte do complexo médico-hospitalar UNIFESP-Hospital São Paulo, que serve como referência médico-ambulatorial e hospitalar para toda a Grande São Paulo, o Estado de São Paulo e outras unidades federadas.

No CERESO, o sistema de atendimento ao usuário é determinado através de uma rotina e de um fluxograma interno, com funcionamento de 10 horas diárias, sendo que o usuário recebe o atendimento oftalmológico sem agendamento prévio, no mesmo dia, seguindo o critério de ordem de chegada.

De acordo com este critério, os pacientes da fila eram triados e até que fosse atingido um número total de 40 usuários em condições de receber o atendimento do CERESO, distribuindo-se 20 pela manhã e 20 à tarde. Os usuários que eventualmente ultrapassassem este corte, eram orientados a retornar ao serviço em outro dia para ser atendido e submeterse novamente a este fluxo podendo até, pelo sorteio aleatório, fazer parte da amostra.

Para a definição do universo e da amostra, utilizou-se como referência os parâmetros médico-assistenciais do manual de Definição dos Indicadores e Parâmetros Médico-Hospitalares da Fundação Hospitalar do Distrito Federal -1986 e da Portaria MPAS 3046/82 do Ministério da Previdência e Assistência Social, e da resolução SS-106, de 27-06-89 publicado no D.O.E. sec. I, São Paulo, 99 (118), quarta-feira, 28-06-89(10). Temos, segundo as referências acima, 22 dias úteis no mês, 264 dias úteis no ano, sendo 4 consultas médicas/hora/profissional (recurso humano). Temos, ainda, a capacidade física de 4 consultas médicas/hora/consultório e o número de consultas médicas por especialidade igual à população $\mathrm{x}$ índice (este índice se refere ao fator de distribuição das consultas médicas especializadas seguindo a portaria MPAS 3046/82 oftalmologia $=0,078$ ).

Porém, em decorrência da presença do profissional da área de Tecnologia Oftálmica que auxiliaria o oftalmologista na realização de alguns exames prévios à consulta oftalmológica, utilizamos como referência, 20 consultas/período/profissional i.é, 5 consultas/hora ao invés de 16 consultas que seguiriam a normatização descrita. Os recursos humanos que fizeram parte deste serviço incluem, por jornada de 4 horas, 1 médico oftalmologista, 1 profissional da área de tecnologia oftálmica, 1 enfermeira, 1 auxiliar de enfermagem, 1 escriturário, 1 servente e 1 segurança.

O fluxo de atendimento interno do CERESO, as responsabilidades e atribuições de cada funcionário e a orientação do preenchimento dos formulários foram descritos e normatizados em um manual de operações.
Para definição da nossa amostragem, foi realizado sorteio aleatório de um dia útil de cada semana - a não ser semanas previamente com feriados da instituição -, entre os meses de fevereiro a julho de 1998, sendo realizado um cronograma mensal de atendimento dos médicos oftalmologistas, cronograma esse de domínio apenas dos pesquisadores padronizados para se evitar possíveis tendências e vícios. Caso o dia sorteado do atendimento fosse feriado religioso, cívico ou similar, $\mathrm{o}$ atendimento seria realizado no dia útil que o antecedesse.

Inicialmente fez-se o levantamento da produção das consultas oftalmológicas, por dia útil sorteado neste período, estabelecendo-se um total de 482 usuários do sistema (nem sempre obtivemos os 40 pacientes/dia para o atendimento). A partir daí coletou-se uma amostragem aleatória referente à aproximadamente $49,4 \%$ (238 usuários) que iriam se submeter ao preenchimento do questionário referente à resolutividade $\mathrm{e}$ satisfação. Desta amostragem faziam parte tanto usuários do horário da manhã como da tarde.

As informações foram coletadas através de questionário aplicado (quadro 1) no próprio centro de referência por um profissional treinado - após o término da consulta oftalmológica e no dia de atendimento -, utilizando-se técnicas para coleta de depoimentos pessoais qualitativos ${ }^{(11)}$.

Estes dados visavam analisar qualitativamente o atendimento recebido pelo usuário. Portanto, foi um estudo de análise qualitativa tendo como referencial o cliente e não o funcionamento do sistema de saúde ${ }^{(12)}$.

Neste questionário priorizamos as seguintes variáveis:

- Identificação: informações destinadas a identificar a clientela, nome do informante e identificação do pesquisador.

- Motivo da procura ao serviço: essa informação tinha como objetivo principal identificar o motivo pelo qual o cliente procurou o serviço, sendo que o critério para agrupamento das respostas foi secundário às respostas obtidas, pois as variáveis não poderiam ser conhecidas anteriormente na sua totalidade. Estes critérios foram os seguintes:

- menção pelo paciente da utilização ou necessidade do uso de óculos;

- referência de sintoma/sinal não relacionado à visão;

- referência à baixa acuidade visual;

- inespecíficos ou encaminhados.

- Resolutividade: essa informação tinha o objetivo de avaliar o resultado do atendimento realizado pelo CERESO frente ao motivo da procura, fazendo-se uma análise qualitativa do mesmo.

- Satisfação: essa informação tinha o objetivo de indicar a satisfação das expectativas do sujeito da atenção (paciente). Em sua maioria: acesso geográfico; oportunidade em tempo de espera na consulta, assim como o tempo necessário para estabelecer o diagnóstico e indicar o tratamento; cortesia; solução do problema médico que motivou a demanda de serviço.

- Utilização deste serviço: essa informação visa identificar a maneira de utilização deste serviço pela clientela usuária e envolvia as seguintes variáveis: 
a- proximidade do local da residência;

b- atendimento no mesmo dia;

c- gostar da UNIFESP;

d- ser paciente de outro departamento da UNIFESP;

e- ser indicado por alguém;

f- outros.

- Número necessário de vezes para ser atendido: essa informação tem como objetivo principal analisar o acesso ao serviço de saúde pela clientela.

- Quantas vezes foi atendido pelo CERESO: visa obter informação sobre a resolução do motivo da procura ao serviço e da necessidade de retornar ao mesmo.

\section{RESULTADOS}

Dos 238 usuários da nossa amostragem, 2 (0,84\%) deixaram de responder ao questionário por apresentarem distúrbios auditivos e mentais e $2(0,84 \%)$ por evasão, sendo estes 4 excluídos da amostra, finalizando 234 usuários que se submeteram à aplicação do questionário.

Com relação ao motivo de procura do serviço pelo cliente obteve-se:

- $106(45,3 \%)$ problemas relacionados ao uso de óculos (dificuldade para enxergar longe, dificuldade para enxergar perto, vista cansada, querer trocar óculos);

- $70(29,9 \%)$ problemas relacionados a sintomas ou sinais oculares (olho vermelho, purgação, lacrimejamento, ardor, dor nos olhos, dor de cabeça, outros sinais ou sintomas);

- $27(11,5 \%)$ problemas relacionados à baixa visão, não especificados;

- $11(4,7 \%)$ outros problemas oculares variados/catarata, infecção;

- $20(8,6 \%)$ outros/inespecíficos ou encaminhamento.

Em relação à questão $n^{0} 1$ e n 2 , "se o CERESO resolveu ou não o problema que foi motivo de procura pelo cliente", tivemos $201(85,96 \%)$ respostas "Sim" e $33(14,1 \%)$ respostas "Não". Destes 33 que referiram que "o CERESO não resolveu o problema", todos (100\%) foram encaminhados para outro setor do Departamento de Oftalmologia da UNIFESP, não sendo nenhum deles encaminhado a outras instituições.

Quanto à "satisfação com o atendimento do pessoal do CERESO" (questão n 3 ) observou-se que 234 (100\%) da clientela mostrou-se satisfeita, sendo que os principais fatores de satisfação identificados estavam relacionados a "tratamento", decorrente da relação interpessoal com todos os profissionais envolvidos no atendimento, ao exame oftalmológico detalhado, à rapidez do serviço, à educação e à simpatia dos funcionários, à limpeza, à aparelhagem utilizada, à explicação de tudo o que está acontecendo e outros. Encontrou-se, porém, 2 usuários que apresentaram a ressalva da demora do atendimento.

Quanto à resposta "por que usa este serviço? (CERESO)" obtivemos 296 respostas referentes às assertivas apresentadas, sendo que $20(6,8 \%)$ optaram pela assertiva "a"; 63 $(21,3 \%)$ optaram pela "b"; 41 (13,9\%) optaram pela "d". Por questões estatísticas analisamos as respostas "c", "e" e "f" agrupadas, já que estas últimas poderão resultar em respostas subjetivas, necessitando descrição mais detalhada, o que interferiria em nossa análise final. Deste modo, 172 (58,1\%) optaram pelas assertivas "c", "e" e "f".

Obtivemos, também, 57 associações de respostas sendo 9 $(15,8 \%)$ de respostas "b" e "c"; $9(15,8 \%)$ de respostas "b" e "f"; $8(14,0 \%)$ de respostas "b" e "e", $4(7,0 \%)$ de respostas "a" e "b" e outras associações descritas no Gráfico n 1 .

Quanto à resposta $n^{0} 5$ "quantas vezes o $\mathrm{Sr}$.(a) necessitou vir ao CERESO até conseguir atendimento?" obtivemos entre as 234 respostas, $200(85,5 \%) 1 \mathrm{vez} ; 29(12,4 \%) 2$ vezes; 5 $(2,1 \%) 3$ vezes.

Já em relação à questão $\mathrm{n}^{0} 6$ "quantas vezes o Sr.(a) foi atendido(a) pelo CERESO?" obtivemos $213(91,0 \%)$ de respostas $1 \mathrm{vez}, 21(9,0 \%)$ de respostas 2 vezes, totalizando as 234 respostas computadas.

\section{DISCUSSÃO}

Para a análise da resolutividade e da satisfação, a metodologia adotada preocupou-se em verificar se o CERESO conseguiu resolver o principal motivo de procura do serviço pela clientela, ou se houve a necessidade de encaminhamento do usuário para outro setor do Departamento ou a outras Instituições. Considerou-se, também, outras variáveis como:

- satisfação da clientela no atendimento recebido da equipe de saúde, visando analisar a possível relação entre resolutividade e a satisfação apresentada;

- utilização do serviço por questões geográficas, operacionais e econômicas no intuito de investigar o acesso ao serviço.

- tempo dedicado pelo usuário para a resolução do seu problema e para avaliar a otimização do serviço.

A multidimensionalidade destas variáveis dificulta este tipo de análise frente ao atendimento recebido pela clientela ${ }^{(13)}$. Desta forma, a presente investigação optou por abordar a satisfação da clientela do ponto de vista do relacionamento com a instituição e dos profissionais de saúde.

Os dados referentes à aplicação do formulário de resolutividade e satisfação à clientela do CERESO mostram os problemas relacionados ao uso de óculos como o principal motivo da procura ao serviço. Este dado é coincidente com o perfil de morbidade na qual a refração ocular constitui a primeira causa de consulta ocular na Região das Américas ${ }^{(3,8)}$. Sintomas e sinais como ardor, lacrimejamento e cefaléia, entre outros, que representaram $29,9 \%$ das queixas destes usuários e muitas delas apresentavam-se de maneira vaga, poderiam também estar relacionadas com os problemas refracionais ${ }^{(2,14)}$.

Observou-se $11,5 \%$ das queixas relacionadas aos problemas de baixa de visão podendo ou não estarem associadas a causas mais definidas como catarata, retinopatia diabética e outras.

Apenas 4,7\% dos entrevistados associaram o motivo da procura a uma doença ocular específica, sendo que, se confirmássemos a causa principal da baixa de visão, esta prevalência poderia ser maior. 


\section{UNIVERSIDADE FEDERAL DE SÃO PAULO - UNIFESP ESCOLA PAULISTA DE MEDICINA - EPM HOSPITAL SÃO PAULO - HSP DEPARTAMENTO DE OFTALMOLOGIA - CERESO}

\section{QUESTIONÁRIO (resolutividade e satisfação)}

№ Pesquisa:

Data:

Paciente:

Informante:

Pesquisador:

Motivo da procura:

1. O Cereso resolveu o problema? (respondido pelo pesquisador) $\square \operatorname{Sim}$

$\square$ Não

2. Se a resposta anterior for negativa: Por que?

a) Foi encaminhado para outro setor do Departamento

b) Foi encaminhado a outras Instituições

c) Outras: (especificar)

3. Ficou satisfeito com o atendimento do pessoal do Cereso?

a) $\mathrm{Sim}$

b) Não

Por que?

4. Por que usa este serviço? (Cereso)
a) Porque é perto de casa
b) Porque tem chance de ser atendido no mesmo dia
c) Porque gosta da UNIFESP
d) Porque já é paciente de outro Departamento da UNIFESP
e) Por indicação. De quem? Por que?
f) Outros (especificar):

5. Quantas vezes o Sr. (a) necessitou vir ao Cereso até conseguir atendimento?
a) 1 vez
b) 2 vezes
c) 3 vezes
d) 4 vezes
e) Ou mais

6. Quantas vezes o Sr.(a) foi atendido(a) pelo Cereso?
a) 1 vez
b) 2 vezes
c) 3 vezes
d) Ou mais

Quadro 1 - Avaliação da resolutividade e satisfação

Com relação ao grau de resolutividade do CERESO (questão $n^{0} 1$ e questão $n^{0} 2$ ) obteve-se 201 respostas afirmativas $(85,9 \%)$, analisadas em função do fator de deliberação do motivo principal da procura do serviço de saúde ocular. Nesta análise avaliou-se a expectativa do paciente em relação à sua queixa principal se foi satisfeita ou não no momento e no dia da consulta, visto que não foi realizado um seguimento no pós-consulta. Deste modo obteve-se $85,9 \%$ de resolução referente a essa demanda, valor este considerado bom, já que neste nível de atenção oftalmológica, têm-se recursos huma- nos, materiais e físicos próprios deste segmento, capacitandoo à resolução de problemas oftalmológicos que constituem a maior demanda na população e que não necessitam de procedimentos de maior complexidade.

Em vários países a freqüência das necessidades oftalmológicas no nível primário e secundário tem sido reconhecida, e uma atenção oftalmológica neste nível é realizada mediante a integração do oftalmologista à equipe médica de nível primário ou à prestação de horas de serviço no nível primário pelo oftalmologista, ou pelo menos a formação de pessoal auxiliar a quem se delega as funções mais simples do oftalmologista ${ }^{(3)}$.

Com relação à satisfação com o atendimento pelo pessoal do CERESO obteve-se a maioria absoluta dos entrevistados satisfeitos com o atendimento (100\%).

Ao lado das medidas objetivas, cada vez mais sofisticadas e complexas, envolvendo múltiplos indicadores e variáveis, a representação e a subjetividade dos pacientes sobre seu atendimento vêm sendo crescentemente incorporadas a essa análise ${ }^{(15)}$.

A percepção dos usuários do CERESO em relação aos serviços prestados não apresentou novidades em relação a estudos anteriores ${ }^{(12,15)}$.

Segundo Pérez, Ibarra ${ }^{(16)}$, a avaliação da satisfação da população com os serviços de saúde entram na classificação de investigação avaliativa acumulativa e se caracteriza porque seus aspectos se centram num aspecto subjetivo valorativo da população objeto dos serviços, políticas e programas de saúde.

De acordo com este mesmo autor, dentro das investigações avaliativas, a pesquisa feita por avaliador que se encontra em grau direto de dependência com o sistema constitui um erro fundamental. Deste modo, os investigadores de formulário devem ter o máximo de independência de todos os aspectos possíveis relacionados ao sistema objeto de estudo. Esta variável pode, provavelmente, ter interferido diretamente nas respostas referentes à questão da satisfação, já que o entrevistador, apesar de locado em sala que garantia total privacidade, atuava diretamente no fluxograma interno do CERESO. No entanto, os dados obtidos corroboram com outros achados da literatura ${ }^{(16)}$.

Segundo Ramirez et al. ${ }^{(17)}$, o grau de satisfação da clientela é influenciado principalmente pelo acesso social, cultural e geográfico; oportunidade em tempo de espera na consulta, hospitalização e estudo de laboratório, assim como o tempo necessário para estabelecer o diagnóstico e indicar o tratamento; cortesia; solução do problema médico que motivou a demanda de serviço; informação sobre a natureza da enfermidade, sobre o controle e a prevenção das complicações, sobre o tratamento e outras medidas gerais; e, finalmente, as comodidades como iluminação adequada, ventilação, ordem, limpeza e elementos e situações importantes do meio ambiente que se esperam durante o processo da atenção médica.

As principais variáveis identificadas referiam-se a relação interpessoal com os profissionais envolvidos no atendimento, além da questão concernente à tecnologia, à rapidez do serviço, à limpeza e às orientações em todos os níveis do atendimento - tanto sobre o fluxo de atendimentos como dos problemas oculares encontrados. 
Os aspectos mais influentes na satisfação do paciente são: a informação do mesmo sobre sua enfermidade e seu tratamento; a relação que o médico consegue estabelecer com ele; o critério que o paciente tem sobre a competência do médico e, com menor peso, as condições de tratamento tanto físico como culturais, além da coerência do trabalho da equipe de saúde ${ }^{(16)}$.

Só 2 usuários mencionaram a demora do atendimento provavelmente por serem pacientes que tiveram que aguardar $\mathrm{o}$ atendimento no período da tarde -, o que talvez justifique esta resposta.

Das 296 respostas para a questão $n^{0} 4$ "por que usa este serviço?" (gráfico 1), com relação às assertivas analisadas individualmente, o maior número de respostas $(21,3 \%)$ foi ter procurado o CERESO pela oportunidade de ser atendido no mesmo dia, seguido da assertiva "por ser paciente de outro Departamento da UNIFESP" $(13,9 \%)$ e, finalmente, "por ser perto de casa" $(6,8 \%)$. Destacamos que houve um número maior de respostas analisadas (296) em relação ao número dos usuários (234), pois o cliente optou por mais de uma assertiva.

Estes dados evidenciam a necessidade do estabelecimento de um serviço de atendimento que otimize tempo e espaço, que viabilize custo e benefício já que, nestas respostas, observou-se a prioridade do usuário em ser atendido no mesmo dia - o que implicaria em menor gasto com transporte e menor perda de tempo do horário de serviço. A utilização de serviços próximos aos que os usuários normalmente estão acostumados a utilizar e a proximidade do local de moradia são variáveis a serem consideradas também. São importantes pois este serviço se situa em uma cidade cosmopolita que apresenta grandes dificuldades de acesso ao transporte por parte da população.

Com a análise agrupada das assertivas "c", "e" e "f" tivemos 172 respostas $(58,1 \%)$. Com relação às demais assertivas, estas foram agrupadas por se incluírem em um grupo de respostas subjetivas como "gostar da UNIFESP", "indicação porque", obtendo-se respostas muitas vezes não explícitas e detalhadas, dificultando a análise individual de cada grupo.

Analisando-se a assertiva "c" "por que gosta da UNIFESP", pode-se interpretar a afirmativa tanto pela qualidade do serviço como pela facilidade de localização do mesmo.

Em relação à assertiva "d" "porque já é paciente de outro Departamento da UNIFESP" a análise é mais complexa, pois não se pode afirmar que a procura ao serviço foi em decorrência do usuário já estar habituado ao sistema (o que facilitaria o seu acesso), ou se foi pela escolha da qualidade do mesmo.

Por questões operacionais e equacionando-se custo/benefício, a assertiva "b" é de fundamental importância na análise da utilização deste serviço, o que corrobora com as respostas obtidas no formulário, pois das demais assertivas associadas, a "b" estava presente em todas elas $(15,8 \%$ associada com "c", 15,8\% com "f", 14\% com "e", 7\% com "a").

$\mathrm{Na}$ análise da resposta número 5 - que se refere ao número de vezes necessárias para se conseguir o atendimento no CERESO -, na grande maioria $(85,5 \%)$ obteve-se a necessidade de 1 vez. Isto estaria revelando, de um modo geral, o acesso ao serviço. Certamente os usuários que necessitaram retornar para serem atendidos não tiveram suas expectativas atendidas em um primeiro momento porém, poderiam pelo sorteio aleatório fazer parte de nossa amostra e responderem ao questionário aplicado. Se tivéssemos tido uma porcentagem significativa de pacientes que necessitaram retornar duas ou mais vezes para serem atendidos implicaria na necessidade da ampliação dos recursos físicos e humanos, a fim de se otimizar a capacidade instalada deste ambulatório.

A investigação do número de vezes que o usuário foi atendido pelo CERESO (questão 6) permitiria analisar se o motivo das visitas subseqüentes foi resultante da não resolução do motivo da primeira visita. Porém a assertiva proposta foi inconclusiva por não especificar se os $9 \%$ dos retornos ao CERESO foram devidos a outro motivo que não o principal.

\section{CONCLUSÃO}

A clientela demonstrou alto grau de satisfação pelo atendimento recebido.Os principais motivos de procura dos usuários ao serviço foram resolvidos. A organização dos serviços oftalmológicos, com a implantação de referência secundária, teria certamente impacto positivo na solução de problemas oftalmológicos - especialmente os refracionais. Deste modo, esta organização amenizaria um importante problema de saúde pública.

\section{ABSTRACT}

In developing countries blindness prevalence could be reduced if access to ophthalmic assistance for the population were not impaired by the lack of organization of the resources.

Besides the primary level, organization of ocular care establishes secondary and tertiary care levels, according to the complexity of the diagnosis and treatment of the ocular diseases.

The Ophthalmic Secondary Reference Center (CERESO) of the Department of Ophthalmology, Federal University of São Paulo, according to an internal flow model of assistance, selects cases of low complexity to be seen on the same day, the others being referred to the tertiary level.

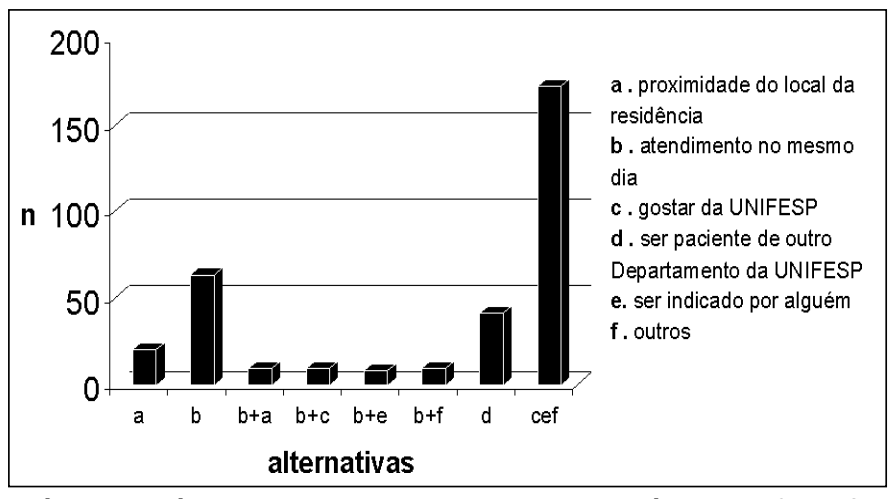

Gráfico 1. Número de respostas dadas por usuários do CERESO UNIFESP/EPM à questão "Por que usa este serviço?", em 1998 
Purpose: To evaluate CERESO's resolution and its user's satisfaction. Methods: By means of applying a questionnaire based on the resolution of the main complaint and for the client's satisfaction, a sample of 238 users was interviewed from February to July, 1998. Results: CERESO's resolution degree was $85.96 \%$; the user's satisfaction was $100 \% ; 6.8 \%$ of the users chose CERESO because it was close to their homes; $21.3 \%$ because they could be seen on the same day; $13.9 \%$ because they were already UNIFESP patients and $58.1 \%$ because they like UNIFESP or were indicated by another patient. Conclusions: The customers were very pleased with the assistance they were given, and their major aims were achieved. The organization of the ophthalmological services, with the secondary care service establishment, would certainly have a positive impact on the solution of ophthalmic problems - especially refractive errors. For this reason, this organization would case an important public health problem.

Keywords: Blindness/prevention \& control; Health care levels; School health; Outcome and process assessment (health care); Health care quality assurance; Patient satisfaction; Health services; Delivery of health care

\section{REFERÊNCIAS}

1. Thylefors B, Négrel AD, Pararajasegaram R, Dadzie KY. Global data on blindness. Bull World Health Organ 1994;73:115-21.

2. Carazo V, Rico Velasco J, Beltranena F, Quesada T. Guias para gerencia de servicios de atencion ocular. Washington, DC: Organizacion Panamericana de la Salud; 1986. (COLL/PNSP/86/101).

3. Simeant SJ. Estudio de la capacidad resolutiva de la demanda em atención de morbidad a nivel primário (área urbana). Cuad Méd Soc (Santiago de Chile); 1983;24:156-69.

4. Donabedian A. La dimensión internacional de la evaluación y garantia de calidad. Salud Pública Méx 1990;32:113-7.

5. Aguirre-Gas H. Evaluación de la calidad de la atención medica. Expectativas de los pacientes y de los trabajadores de las unidades medicas. Salud Publica Méx 1990;32:170-80.

6. Castellanos J, Robayo J. Situaciones de cobertura, niveles de atención y atención primaria. Bol Oficina Sanit Panam 1977;82:478-92.

7. Organización Panamericana de la Salud. Programa de Investigación y Desarrollo de Sistemas de Salud. Servicios de atención médica ambulatória: se usan en la forma apropiada? Bol Oficina Sanit Panam 1981;90:388-409.

8. Carazo V, Rico Velasco J, Beltranena F, Quesada T. Evaluating the conditions of efficiency. In: Carazo V, Rico Velasco J, Beltranena F, Quesada F. Guías para gerencia de servicios de atención ocular. Washington, DC: Organizacion Panamericana de la Salud; 1986. (COLL/PNSP/86/101).

9. Campero-Cuencas L. Calidad de la relación pedagogica medico-paciente en consulta externa: el caso de Oaxaca, Mexico. Salud Publica Méx 1990;32: 192-204.

10. Brasil. Ministério da Saúde. Conselho Nacional de Saúde. Publicação 3046/ 82. Resumo executivo da centéssima décima oitava reunião ordinária do CNS. Brasília: Ministério da Saúde; 1982 [citada 2002 Jan 9]. Disponível em URL: http://conselho.saude.gov.br/docs/ResumoExecutivo_ata118.doc

11. Queiroz MI. Histórias de vida e depoimentos pessoais. Sociologia 1953;15:9-24.

12. Tanaka OU. Avaliação da utilização dos serviços do Posto de Assistência Médica e do Posto de Atendimento do Jardim São Jorge, no Município de São Paulo [tese], São Paulo, Faculdade de Saúde Pública da Universidade de São Paulo; 1983.

13. Linder-Pelz S, Struening EL. The multidimensionality of patient satisfaction with a clinic visit. J Commun Health 1985;10:42-54.

14. Brasil. Ministério da Saúde. Secretaria de Assistência à Saúde. Coordenação de Doenças Crônico-Degenerativas-CODEG. Informações básicas para a promoção da saúde ocular. 2.ed. Brasília: Ministério da Saúde; 1994.

15. Siqueira MM. Eqüidade na saúde: uma análise em serviços ambulatoriais públicos em Belo Horizonte. Rev Adm Pública 1990;24:53-9.

16. Pérez R, Ibarra AM. La evaluación de la satisfacción de la población con los servicios de salud. Rev Cub Adm Salud 1985;11:255-62.

17. Martinez Ramirez A, Van-Dick Puga MA, Nápoles Rodrigues F, Robles Uribes J, Ramos Ramos A, Villaseñor Urrea I. Hacia una estrategia de garantía de calidad: satisfacción en la utilización de los servicios médicos. Cad Saúde Pública 1996;12:399-403.

\title{
Encontro Anual da Association for Research in Vision and Ophthalmology - ARvo
}

\author{
4 a 9 de Maio de 2003
}

Fort Lauderdale - Flórida - EUA

\author{
INFORMAC̣ÕES: E-mail: arvo@arvo.org \\ Home page: www.arvo.org
}

